\title{
The use of 'think aloud' technique, information processing theory and schema theory to explain decision-making processes of general practitioners and nurse practitioners using patient scenarios
}

Maxine Offredy Faculty of Health and Human Sciences, University of Hertfordshire, Hatfield, UK and Elizabeth Meerabeau School of Health University of Greenwich, Eltham, UK

\begin{abstract}
This paper focuses on one aspect of a research study exploring the cognitive processes of decision making by 11 nurse practitioners and 11 general practitioners from the south east of England, using six patient scenarios during 2000. It sets out to explain some of their decision-making processes. This paper is part of a larger study discussing the use of information processing theory as a framework for exploring decision-making. Schema theory is used to provide explanation of correct and incorrect responses to the six scenarios. The paper explores areas in which cognitive overlap occurs and uses the participants' examples to illustrate schema functioning. The usefulness of information processing theory to explore decision-making by nurse practitioners is also addressed. The paper concludes by suggesting that information processing theory and 'think aloud' approaches were suitable for identifying errors in decision-making, and could therefore be used as a teaching tool. Change is required in the culture of primary care organizations if shifting emphasis towards developing collective responsibility and greater openness is to be achieved.
\end{abstract}

Key words: cognitive processes; decision-making; information processing theory; nurse practitioners; schema theory

\section{Introduction}

The 'think aloud' approach to the study of decision-making was developed from information processing theory (Newell and Simon, 1972) and requires participants to think aloud as they solve either real or hypothetical problems. Schema theory describes the construction of mental models for problem-solving. This method was chosen for this study as the process allows researchers to gain an understanding of the "paradox of expertise' (Johnson, 1983), by analysing the transcripts of the tape-recorded verbalizations. Johnson

Address for correspondence: Maxine Offredy, University of Hertfordshire, Faculty of Health and Human Sciences, College Lane, Hatfield, Herts, AL10 9AB, UK. Email: M.V. Offredy@herts.ac.uk
(1983) explained that while it is important to know how experts think so that others may be helped to think in a similar way, experts are usually unable to provide detailed descriptions of how they perform tasks.

The discussions in this paper form part of a larger qualitative study, conducted in 2000, which explored decision-making consultations in primary health care by nurse practitioners and general practitioners for diagnosis and treatment when given the same patient scenarios. This paper provides explanation of the decision-making processes of the two groups of participants. Schema theory is used as the basis for the explanation, whilst the usefulness of information processing theory as a framework for exploring decision-making is examined. The paper begins by briefly outlining the aims and methods of the larger study, thereby contextualizing the discussion of this article. It then introduces 
schema theory before explaining the reasons for correct and incorrect responses to the scenarios summarized in Table 1.

\section{The study}

\section{Aim}

The aim of the larger study was to ascertain the differences, if any, in the decision-making processes of nurse practitioners (NPs) and general practitioners (GPs) for diagnosis and treatment when given the same patient scenarios. This paper provides an explanation of the decision-making processes of the two groups.

\section{Methods}

A summary of study procedures will be provided here as full details of this have been reported elsewhere (Offredy, 2002). Twenty-two participants, 11 NPs and 11 GPs, from the south east of England, chosen by purposeful sampling took part in the main study following the pilot. The two inclusion criteria for the NPs were that: a) they had to be qualified nurse practitioners who had undertaken the Royal College of Nursing (RCN) nurse practitioner degree programme; and b) their current employment had to be as a nurse practitioner in a primary care setting working alongside a GP. The single inclusion criterion for the GPs was employment of a nurse practitioner (as a nurse practitioner) as part of the health care team. Six scenarios were used for study. The presenting situations were: a rash, an unhealed wound to the cheek, back pain, persistent cough, vaginal discharge and a request to register as a new patient with the surgery. The correct diagnoses for these situations were respectively shingles, cigarette burn to the cheek, lower back pain, chest infection, pelvic inflammatory disease and hypertension. These scenarios were chosen because their prevalence in general practice ranged from $9 \%$ (circulatory diseases) to $31 \%$ (respiratory diseases) (McCormick et al., 1995). Two NPs and a GP who were not involved in the study were given the task of choosing the scenarios. A reference model, devised for each scenario, indicated critical and relevant cues. Critical cues were necessary for successful diagnosis of the condition and relevant cues provided potentially important information for reaching an accurate diagnosis (Joseph and Patel, 1990; Offredy, 2002). Each reference model described the signs, symptoms, type of examination or procedure that might be undertaken, as well as the treatment or range of possible options that could be decided. Written information about the research was posted to each participant two weeks prior to the interview. The conditions in the scenarios were not disclosed in the participants' letter. However, explanation was provided about the research question, the aims of the research, the 'think aloud' procedure and that the scenarios used would be usual in general practice. The letter also explained that ethical approval had been obtained for the research and that the identity of the practitioners would be anonymized in the report. Each participant was interviewed individually, in his or her consulting room at the general practice. At the time of the interview, a reminder of the aims of the research was given, as well as further explanation on the 'think aloud' procedure. A different scenario was used with the participants as a 'test run' to familiarize them with the procedure.

\section{Protocol analysis}

The data generated by the 22 participants, each providing verbalizations on six scenarios, were substantial $(N=132$ transcripts). Participants were given a number to protect their identity. The interviews were transcribed verbatim. Computer software, QSR NUD*IST (1997), was used for data management.

Two types of decisions were observed in the transcripts; one was concerned with the cognitive processes of diagnostic decision-making and the other was therapeutic decision-making or treatment. Coding reflected these two separate types of decision-making. For the cognitive mode of decision-making (diagnosis), Elstein et al.'s (1978) four stages of the reasoning process were used, namely: 1) cue acquisition; 2) hypothesis generation; 3) cue interpretation; and 4) hypothesis evaluation. Additional codes were required for the second type of decision-making (therapeutic decisions) and were developed from the analysis of the pilot. The additional codes were 5) diagnosis; 6) treatment; 7) advice; 8) further treatment/advice/refer to outside agency; and 9) refer to GP. Thus, nine stages compose the decisionmaking of the participants. Each of the nine 


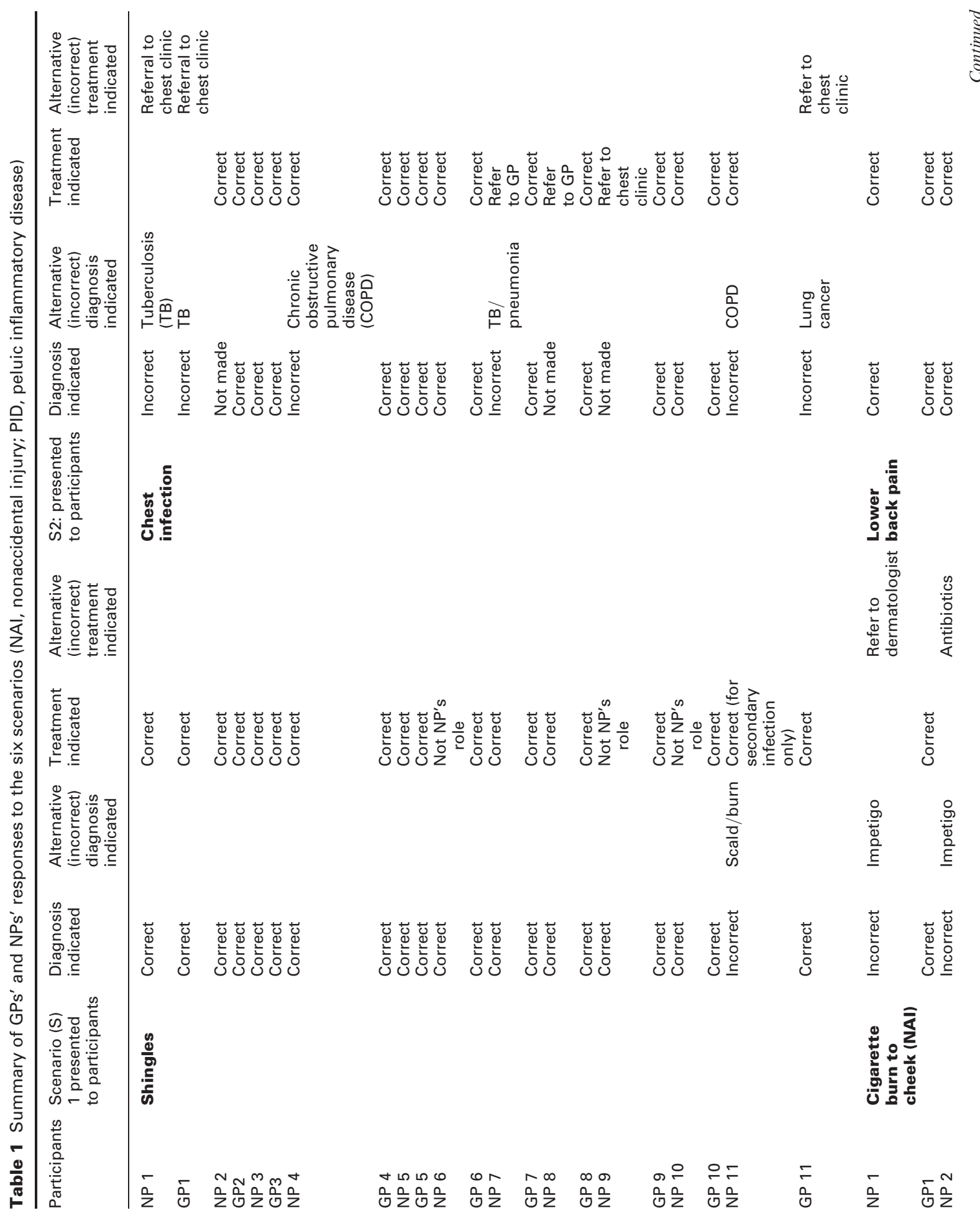




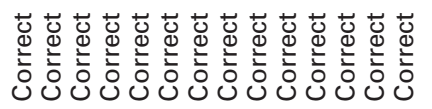

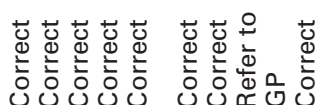
熟譥

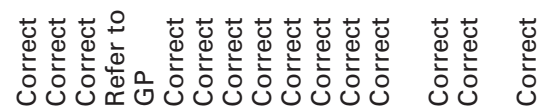

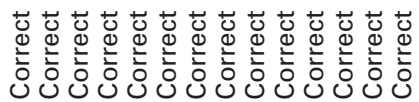

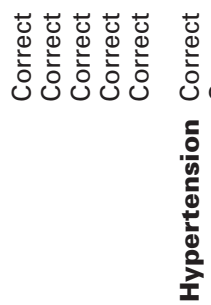
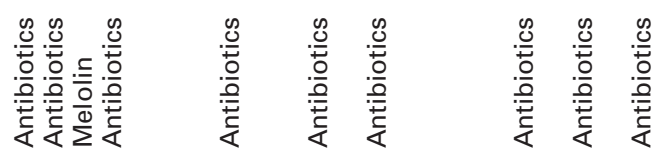

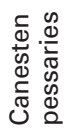
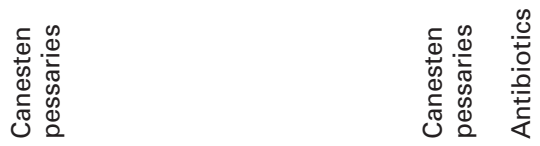

0 $\quad 0$ i

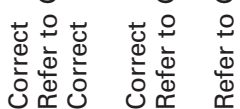

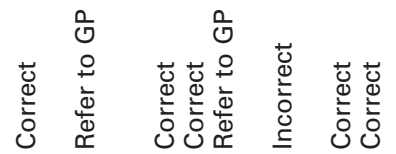

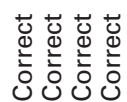

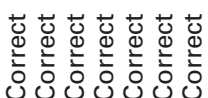

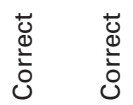

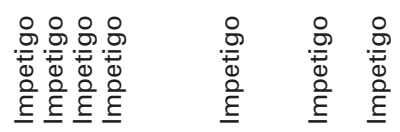

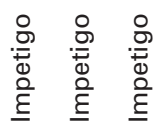

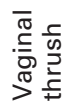

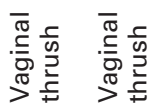

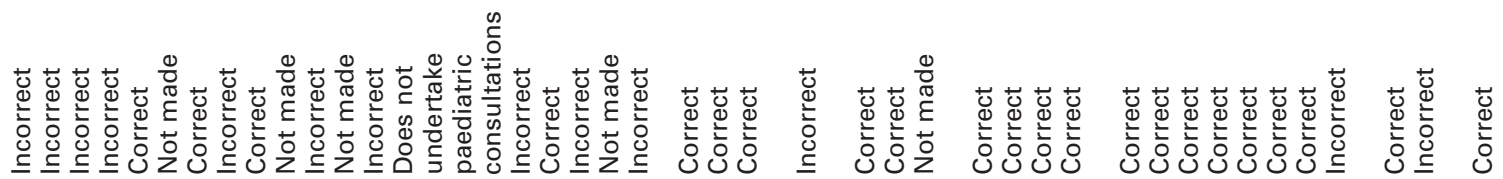

음

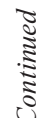

กMm+tนL

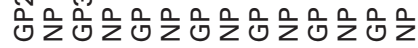

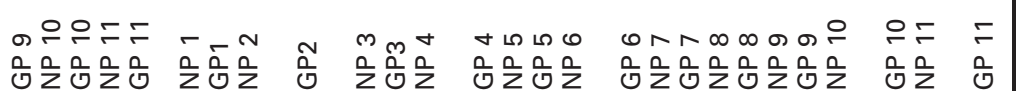
Primary Health Care Research and Development 2005; 6: 46-59 
stages was further subdivided to provide more explanation about the cognitive processes and the decisions made by the participants to conclude the exercise. The following section outlines a brief review of schema theory before providing an explanation of responses to the scenarios.

\section{Schema theory}

The word 'schema' originated from the Greek language meaning 'form', 'shape' or 'figure'. In modern psychological usage schema draws upon the individual's application of knowledge, found in memory, to make sense of experience in their world. The mid-1970s witnessed a resurgence of schema theory by Minsky (1975), Schank (1975) and Rumelhart (1975). Each of these authors provided their own perspective on the use of schema theory with Minsky (1975) proposing that knowledge in human memory can be structured into frames. A frame represents a data-structure of a stereotypical situation, for example, attending one's general practitioner. Attached to each frame are different types of information, which guide behaviour as to what may happen next, or what to do if these expectations do not occur. Minsky (1975) likened frames to a network of nodes and relations. Collections of frames are linked together into frame-systems. In turn, the frame-systems are interconnected in an information retrieval network. When a situation is represented by a frame, a matching process takes place. This is similar to Newell and Simon's (1972) view that knowledge is structured and stored in interrelated chunks in memories of different types and capacities.

Schank (1975) agreed with much of what Minsky (1975) said about frames, and developed the frames idea to lend itself to applications in daily life. Instead of using the word 'frames', Schank (1975) used the concept of scripts to address discourse and comprehension. A script is a predetermined, stereotyped sequence of actions that define a well-known situation. Scripts organize new inputs in terms of previously stored knowledge (Schank and Abelson, 1977). Scripts also describe social events that share common structures between individuals. This implies that individuals can discuss an event because of their similar understanding of that script. Greenwood
(2000) suggested that scripts are similar to action schemata because they represent what is to be done. She stated that:

scripts are the means whereby an agent's overall intention is translated into hierarchically organised instructional units which reflect the structure of the movements required and which eventually issue in action.

Greenwood, 2000: 432.

Rumelhart (1975) retained the word schema in his work on representations in memory. He argued that schemata are structures for organizing data; the implication being that individuals are able to make sense of their world by the structures they create, otherwise each piece of information would be regarded as new and uncategorized. Categorization is a necessity for intelligent action.

Marshall (1995) explained that four types of knowledge are included in schema theory. These are: 1) identification knowledge; 2) elaboration knowledge; 3) planning knowledge; and 4) execution knowledge. Identification knowledge allows recognition of an issue; its key task is pattern recognition. Pattern recognition happens as a result of the many cognitive processes occurring together; no single stimulus acts as a trigger to the recognition. Each schema has its own distinct identification knowledge, although schemata may share some commonalities. Elaboration knowledge contains details about the key elements of the event or situation causing a schema to be developed. In other words, this type of knowledge draws on specific examples from the individual's experience and explanations may be offered relating to the incident. Elaboration knowledge also helps the individual to create a mental picture of the current situation. When the general situation has been recognized by identification knowledge, information about the current experience will be accessed from a 'template' about the situation. Understanding of the current issue depends on how well or badly it fits the schema template. Identification and elaboration knowledge make up a network that allows the individual to construct a working hypothesis about the situation at hand and then to retest it. Planning knowledge refers to the ability to use the schema to be creative, make plans and establish goals and 
subgoals when confronted with situations. This ability is derived from experience. It is possible for individuals to have identification and elaboration knowledge but have no planning knowledge. Thus, they would not have a working schema. The possession of execution knowledge permits the individual to carry out plans. In summary, schemata are data patterns for representing generic concepts stored in memory. They are networks of interrelations that are believed to hold the attributes of the concept that is being discussed. Attributes will vary according to the concept under discussion. It is this variability that gives schema its flexibility to recognize patterns since schemata may exist at different levels of abstraction and complexity. A limitation of schemata is that they fail to address precisely how their components interact.

In their own way the work of Minsky (1975), Schank (1975), Rumelhart (1975) and Marshall (1995) describes the construction of mental models for problem-solving. Constructing a mental model involves recognition of a problem, forming a mental image of it, transforming an initially vague strategy into a structured one, and finally using the strategy to plan and execute a solution. Table 1 summarizes the scenarios used in the study and the responses provided by the GPs and NPs. The following provides a discussion of the differences found in the findings of the scenarios. These are classified under two headings: correct and incorrect responses to the scenarios.

\section{Explanation of correct responses to the scenarios}

According to Marshall's (1995) theory, the study participants were able to identify a situation, event or experience where similar patterns of cues were encountered. Patterns can be visual, auditory or olfactory. They may be acquired through didactic instruction or clinical experience and will range from simple to complex, with concomitant configuration of features. Patterns or cues can also be referred to as forceful features. Gale and Marsden (1985) suggested that forceful features are:

keys to memory, providing the mechanism for gaining access to the information stored there. What acts as a forceful feature depends on the way the person has organised his/her knowledge in the memory store.

Gale and Marsden, 1985: 69

For example, analysis of the chest infection scenario indicated that relevant information might be stored in the clinician's memory, but if it is not organized in a way that is accessible via forceful features that are clinically relevant, it will not be of use when appropriate. Gale and Marsden (1985) maintained that a forceful feature can be made up of any number of pieces of information and may be the 'key' of several memory structures. The skill lies in fitting the forceful feature/s to the appropriate memory structure/s (Figure 1).

Figure 1 demonstrates how forceful features direct the study participants to make new interpretations in the light of new information. When the participants were presented with the scenario of a patient complaining of vaginal discharge, this suggested a normal state. When presented with further information they used the original information and the new data (or cues) to make a different interpretation of vaginal thrush. The same information was reinterpreted in the light of new information (that is, after examination of the patient) to give a diagnosis of pelvic inflammatory disease. Two forms of reinterpretation can be seen: first, reinterpretation of the same information by associating it with different memory structures; and second, reinterpretation of additional information (after patient examination) which gives new forceful features or cues

\begin{tabular}{|l|l|}
\hline Clinical information presented & $\begin{array}{l}\text { Interpretations of forceful } \\
\text { features and associated memory } \\
\text { structures }\end{array}$ \\
\hline Vaginal discharge & $\begin{array}{l}\text { Interpretation 1: Normal } \\
\text { "All women have vaginal } \\
\text { discharge" (GP4). }\end{array}$ \\
\hline & $\begin{array}{l}\text { Interpretation 2: Vaginal Thrush } \\
\text { History of discharge: onset, colour, } \\
\text { odour, type }\end{array}$ \\
\hline $\begin{array}{l}\text { Interpretation 3: Pelvic } \\
\text { Inflammatory Disease } \\
\text { History + patient examination }+ \\
\text { tender lower abdomen + tender on } \\
\text { cervical palpation = Pelvic } \\
\text { Inflammatory Disease }\end{array}$ \\
\hline
\end{tabular}

Figure 1 Clinical information and associated memory structures

Primary Health Care Research and Development 2005; 6: 46-59 
which link to different memory structures so that a new interpretation is made. This iterative process of transforming an initially vague or incomplete schema into one that is well specified is an essential part of problem-solving. Each of Marshall's (1995) four types of knowledge is used to illustrate the participants' responses.

1) Identification knowledge: The cues elicited from the six scenarios where responses were correct triggered many forceful features in the cognitive processes of the practitioners to activate identification knowledge schemata. These forceful features are interconnected elements that form structural representations and are essential for schema knowledge. The degree of connectivity varies in strength and type. Connections may be excitatory or inhibitory (Marshall, 1995). Analysis of the 'think aloud' process revealed that in some scenarios, for example scenarios 1 (shingles), 4 (lower back pain), 5 (pelvic inflammatory disease) and 6 (hypertension), participants utilized specific cues to arrive at a judgement. It could be argued that at this juncture excitatory connections might have triggered some features of elaboration knowledge to enable the participants to build upon their existing schema template. Crucially, the individual must possess sufficient knowledge in order to assess whether the current information being received fits with the previous schema template. Perhaps it is at this stage that differences in the knowledge base of the two groups would reveal themselves since doctors have a broader knowledge base than NPs. Both groups are taught to make differential diagnoses. The differential diagnoses include the most probable or common conditions, as well as the least common conditions that may have adverse consequences if there is a delay in diagnosis. Doctors may have a wider range of differential diagnoses than NPs given their experience and the difference in their education and training, particularly in decision-making.

2) Elaboration knowledge: The 'think aloud' transcripts revealed that not all participants engaged in elaboration knowledge. An experienced practitioner might appear to bypass this process. It would seem that as individuals learn a skill, the repetitious use of the skill requires less effort; it becomes automatic (Baron, 1994). Baron (1994) conceded that automatization is not fully understood and was of the view that it may be a consequence of doing things more quickly so that various demands of the problem can be undertaken in quick succession, or, as practice increases less attention is required to perform the task. Mental patterns gained from experience and fitted in a cohesive and connective way are referred to as analogical reasoning. Analogies are useful in problemsolving if the practitioner is able to conceptualize which elements of the new problem correspond to elements of the old one. Thus, elaboration knowledge is important in analogical reasoning. As cue acquisition increases, the probability of each hypothesis/diagnosis is re-evaluated in the light of the new information - this is a form of hypothetico-deductive reasoning. ${ }^{1}$ As novices gain experience, they become more adept at identifying solutions to analogous problems, which may be applicable in new situations (Parrino and Mitchell, 1989).

Shanteau's (1992) study on the amount and quality of data collected by doctors can be applied to NPs. The study showed that experienced doctors have the ability to distinguish between relevant and non-relevant data and that unnecessary data can be confusing, unhelpful or are sometimes dangerous. An earlier study by Norman and Tugwell (1982) found that thoroughness of cue acquisition was uncorrelated with attaining the correct diagnosis. Identification and elaboration knowledge form the network that enables the practitioners to make and evaluate their hypotheses. At this stage the practitioners are making a judgement - that the condition

\footnotetext{
${ }^{1} \mathrm{~A}$ scientific hypothesis is a proposed explanation of some natural phenomenon. This hypothesis can be tested because it must make specific predictions which would be the logical consequence of the hypothesis and the experiment if the hypothesis is indeed an accurate description of how nature works. This manner of testing, in which the hypothesis serves as the assumption and predictions as conclusions is known as the hypothesis-deductive method and is associated with the work of Karl Popper (1902-1994).
} 
resembles one of shingles, chest infection, lower back pain or pelvic inflammatory disease. Thus for problem-solving, an important heuristic method is to retrieve from memory a problem in the same domain and to try to relate those cues to the ones of the present problem. In other words, identification knowledge (retrieval from memory) is as important as elaboration knowledge (schema construction). Having made the judgement, the next step is to make a plan of what to do.

3) Planning knowledge: Planning knowledge can be seen as mental flow diagrams indicating what action to take when evaluating a particular complaint or finding, as in scenarios 2 (chest infection) and 6 (hypertension). Elaboration and planning knowledge require the practitioner to think through the situation and, depending on the data gathered, the mental flow diagram can be constructed from repeated use of established practice protocols or national guidelines. For example, some general practice protocols suggested that in some circumstances antibiotics may be given pending confirmation of laboratory results. Such decisions are exemplified in scenarios 2 (chest infection) and 5 (pelvic inflammatory disease) where quick recognition of a pattern and its concomitant response is recommended. The response to these conditions becomes automatic and the time lag between diagnosis of the condition and commencement of therapy is decreased.

4) Execution knowledge: In the case of scenario 6 (hypertension) several readings would be taken over a specified period before a diagnosis of hypertension would be made. This procedure - a type of algorithm - is in line with British national guidelines (Ramsay et al., 1999). With the exception of two NPs, no medication would be given to the patient at their first visit. This, again, is in line with national guidelines. Practitioners can be aware of what to do, but because of, for example, organizational and legal constraints, they were unable to engage in execution knowledge. This was exemplified by NPs 2,6 , 9 and 10 where the NPs referred to the GP because the decision on the treatment of the presenting problem was outside their role (organizational constraint), to confirm the diagnosis, or to obtain a signed prescription (legal constraint). In other words, in the four general practices $(\mathrm{P} 2,6,9$ and 10) the decision of how to manage and treat the patient rested with the GPs. In scenario 1 (shingles), 10 of the 11 NPs were able to discuss their decision of how and why they would treat the patient. In other words, the NPs were utilizing execution knowledge. However, where NPs were unable to recognize salient data they may have had incorrect responses, which are discussed below.

\section{Explanation of incorrect responses to the scenarios}

When an NP does not instantly recognize a problem, the NP will seek to establish further facts about the case, while at the same time holding one or more hypotheses in mind. This knowledge is akin to elaboration knowledge, where the focus of attention of the NP is on the list of competing diagnoses that has been brought about during the clinical encounter. The stored details in identification knowledge act as the recognition requirement so that the practitioner can assess whether his/her schema fits the problem. In some cases, the identification knowledge schema may not be a perfect fit for the problem at hand, because crucial elements of the problem are missing from the schema. The elaboration knowledge provides the shape of the mental model, showing its key characteristics. These key characteristics are derived from the problem. It could be at this point where connections may be inhibited. Marshall (1995: 55) explained that a function of a schema is to provide "where reasonable and appropriate the default characteristics that allow the schema to be used' (Marshall's emphasis). Inaccuracies may arise here as the missing crucial elements are replaced by too many default characteristics. The practitioner may choose to collect more data, scan for other possible schemata or proceed with the current schema in the knowledge that its fit is problematic. This latter point may account for the inaccurate diagnosis of scenario 3 (cigarette burn to cheek - nonaccidental injury) where the majority of practitioners indicated that the condition was one of impetigo, although its resemblance was not 'classic impetigo' (GPs and 
NPs in scenario 3). The assumption is that practitioners reverted to the use of heuristics and in particular availability heuristics. There are similarities with availability heuristics and schema theory in that relational characteristics play a part in the diagnostic processes of both the former and the latter. Differences, however, can be seen in the analysis of verbal protocols, where the ability to isolate relevant from irrelevant material and to make more inferences from relevant material were more readily established, than with the use of schema theory. Kahneman et al. (1982) warned against the use of heuristics as a reliable tool for decision-making, particularly as they may bias the practitioner and interfere with the diagnostic process. This is most likely to occur when the practitioner's judgement is adversely affected by the heuristics of:

- availability (diagnosing from recent case experience, for example GP2 in scenario 5 (pelvic inflammatory disease)

- representativeness (selecting a diagnosis because of surface resemblance, for example, the majority of practitioners in scenario 3 (nonaccidental injury), and

- anchoring (retaining an early hypothesis (vaginal thrush) despite the provision of new data, for example NP11 in scenario 5 (pelvic inflammatory disease).

Further explanation of an incorrect response is provided using NP11's 'think aloud' response to scenario 5 (pelvic inflammatory disease) (Figure 2). Incorrect diagnosis could be due to the NP's inability to access the accurate identification knowledge schema. It is postulated that the NP may not have been previously exposed to the condition and therefore the appropriate schema was not present or activated. In other words, there was no pattern recognition because there was no experiential knowledge on which to draw. It would appear that the NP was unable to create the appropriate mental model of the current problem from her elaboration knowledge, resulting in uncertainty in interpreting the cues presented. A possible explanation for this is the presence of too many default characteristics. Schema theory suggests that when someone incorporates new knowledge into their thinking - depending on how the information is presented

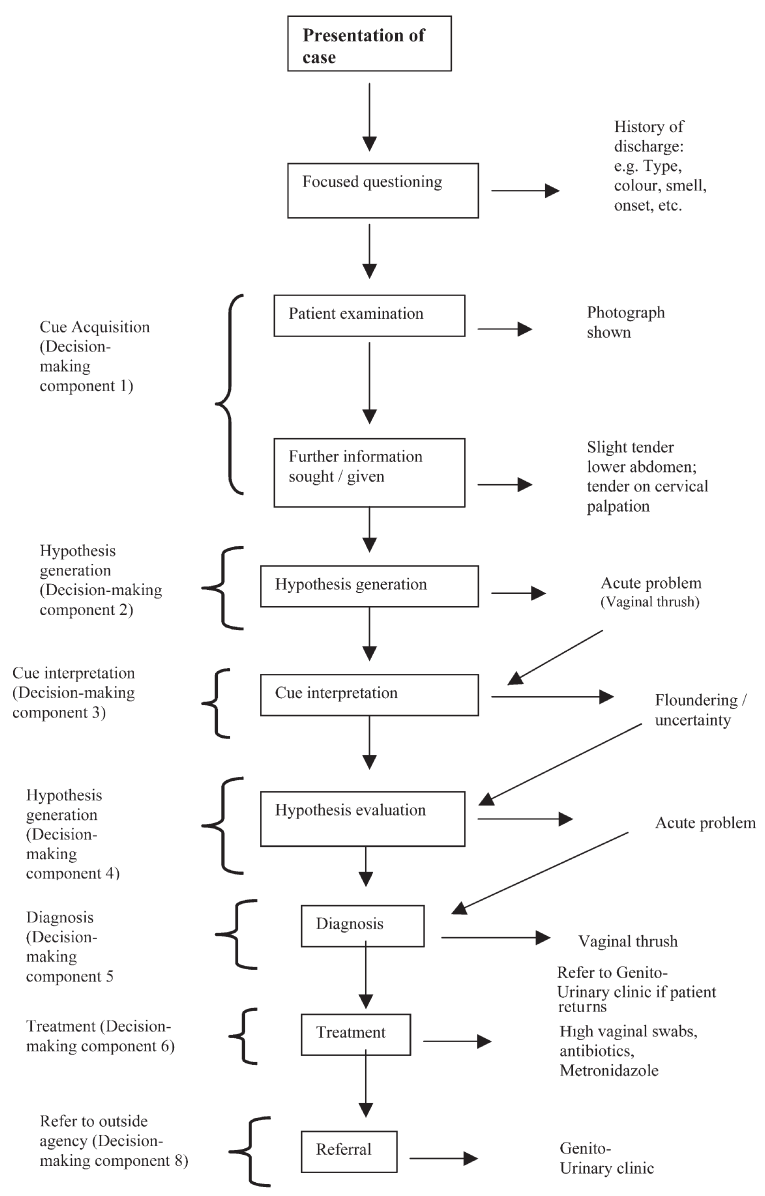

Figure 2 Scenario 5: Pelvic inflammatory disease. Decision-making processes for NP11

- the individual may be able to either (i) work out the general properties of the concept from the specific information provided, or (ii) generate their own example to illustrate the concept from the abstract or basic characteristics of the information given. Marshall (1995) stated that in practice both types of information are usually provided. Thus, schema development is dependent upon the type of information given: strong schemata are more likely to be developed when the new information is presented with an example or a definition of a concept.

Gale and Marsden (1985) pointed out that diagnostic errors may be due to one or more of the following: i) inadequate memory structure, 
ii) inappropriate or inaccessible memory structure, iii) failure to use an appropriate range of thinking processes, and iv) holding on to an incorrect interpretation. Diagnostic performance may be attributed to differences in memory structure and their accessibility rather than only the process of thinking. Gale and Marsden (1985) suggested that differences in 'expertise' might be due to how knowledge and experience are structured and stored in the memory and how they become accessible and usable.

\section{Schema debates}

Although explanation of cognitive processes is divided into four types of knowledge, overlap occurs. The areas in which overlap occurs give rise to two on-going debates.

\section{Conscious versus automatic occurrence of schema}

There is a debate as to whether the individual can consciously and deliberately invoke a schema or whether their use occurs automatically. The answer depends on the presenting problem. If the problem is unfamiliar to the practitioner, identification of the problem implies a conscious and controlled attempt to solve it. Unfamiliar features of a problem will require conscious and deliberate action on the part of the individual. Reason (1990) suggested that when individuals are confronted with a problem, the tendency is to search for a well-tried solution at the rule-based level. That is, the rule is selected from previous successful experiences of problem solving before searching the knowledge-based level (slow, sequential conscious processing), even when the latter is the appropriate route to explore. When individuals persist with incomplete knowledge for problemsolving they tend to match characteristics of the problem to stored problem handling rules: IF (situation) $\rightarrow$ THEN (action) (Newell and Simon, 1972; Reason, 1990). When repeated failure of rule-based reasoning occurs, the practitioner will move to the knowledge-based level of decisionmaking. Reason (1990) commented that even at this stage the problem-solver will try to find cues that are reminiscent of previously successful rules that could be modified to integrate elements of the problem at hand.

\section{Simultaneous versus sequential schema functioning}

The second area of debate is whether schema functioning is simultaneous processing or whether processing occurs sequentially. Marshall (1995) stated that both types of processing are involved in schema production. She explained that simultaneous processing occurs in the recognition of a problem and sequential processing occurs when utilizing planning and execution knowledge. Simultaneous processing refers to networks of small highly related pieces of information that are processed at the same time. The presence or absence of a set of features, together with the ways in which they are linked, determines the recognition of a situation or problem. An example of this is scenario 3 (cigarette burn to cheek, nonaccidental injury). Practitioners developed their own set and subset of features of the condition, which allowed quick recognition of the problem. This was ascertained when the practitioners were asked to explain their reasons for their decisions. Their explanations confirmed elaboration, planning and execution knowledge.

Sequential processing was evident in the verbal protocols of both practitioners in each of the six scenarios. Most pairs of practitioners indicated that they would provide the same treatment/management of the situation, which suggested similarity in the organization and integration of instructional information stored in memory. Their cognitive mechanisms allowed them to map systematic relational structures from one situation to the next. An interpretation of the findings is that the two groups of experienced practitioners have developed knowledge in the form of 'disease scripts' containing prototypical information about diseases. This is similar to Feltovich and Barrows' (1984) concept of 'illness scripts' which the authors described as stereotypical sequential schemata with three main components: i) enabling conditions, ii) faults, and iii) consequences. 'Enabling conditions' are predisposing factors to the presenting problem and include demographic characteristics such as age, sex, occupation, ethnicity and social class. 'Faults' are 
disorders of the presenting problem such as organ failure, trauma and infection. 'Consequences' are the signs and symptoms linked to a particular disorder of the presenting problem. Evidence of the use of enabling conditions in this study was seen, for example, in scenario 1 where 'enabling conditions' (together with the photograph of the condition) were presented to the NPs and GPs. With only this background information, the more experienced practitioners (those with more than two years' NP experience) were more quickly able to arrive at an accurate diagnosis and treatment/management options than was the less experienced practitioner (NP11, eight months' NP experience). This suggests that the experienced practitioners in making their judgements used only information relating to 'enabling conditions' as opposed to using all three components of an 'illness script'. Scenario 3 is another example of using only 'enabling conditions' information to arrive at an accurate diagnosis. Fewer practitioners were able to arrive at a correct diagnosis for this scenario than for scenario 1 .

The findings of scenarios 1 (shingles) and 2 (chest infection) showed that cue activation for these 'disease scripts' led quickly to accurate diagnoses and treatment/management decisions. The proposed explanation is that the prototypical knowledge structure in memory, based on experience with a collection of similar cases, enables patterns of relevant facts to be quickly identified and irrelevant ones to be filtered out. However, practitioners' schemata not only facilitated pattern recognition, they also enabled a coherent and efficient testing procedure for discriminating among competing diagnoses in the decisionmaking process. The experienced practitioner (NP or GP) will know what factors can be left out of the diagnostic equation, whereas those with less experience may not know how to filter out inappropriate or redundant information. These cognitive activities reflect exposure to a wide variety of cases, thereby building up practical experience. This needs to be supplemented with on-going research-based evidence to underpin practice.

The relevance of both these areas of debate (that is, whether an individual can consciously and deliberately invoke schemata or whether their use occurs automatically; and whether schema functioning is simultaneous processing or whether processing occurs sequentially) to the study findings is that both groups of practitioners have similar 'cognitive architecture' (Newell and Simon, 1972). The 'cognitive architecture' explains the way knowledge is stored in human memory and is referred to as the production rule, having the form IF (situation) $\rightarrow$ THEN (action) (Newell and Simon, 1972; Reason, 1990). The decision-making processes of both groups as represented by their verbal protocols show similarities. This means that the practitioners have developed similar rules that govern their skills. Some of these rules have been gained through educational preparation and others have been acquired through experience. Standardized educational preparation and more widespread understanding of the role of NPs would be likely to encourage more GPs to fully utilize NPs' skills.

\section{The usefulness of information processing theory in exploring decision-making by nurse practitioners}

Information processing theory was the framework used in this research to investigate how the practitioners actually think and reach decisions rather than how they should make decisions. The theory emphasizes understanding and explanation of behaviour rather than prediction and control. In using this theory, this study aimed to make a contribution to the understanding and explanation of NPs' decision-making, rather than a contribution to prediction. The use of verbal protocol analysis derived from 'think aloud' procedures allowed explanation to be made of the steps taken to solve problems. The theory posits that hypotheses are used to overcome the limitations of short-term memory, and serve to narrow the size of the problem space. The scope for possible inferences is also narrowed and this in turn limits uncertainty. The generation of hypotheses is a way of restructuring the patient's complaint from: 'What is this patient's illness?' to 'Is the patient suffering from A, B or C?' (Joseph and Patel, 1990). The NPs and GPs using this method concentrated on the cues that were likely to produce an accurate diagnosis. The 'think aloud' protocols provided rich data that approximated to the concurrent reasoning of the NPs and GPs, as well as showing the 


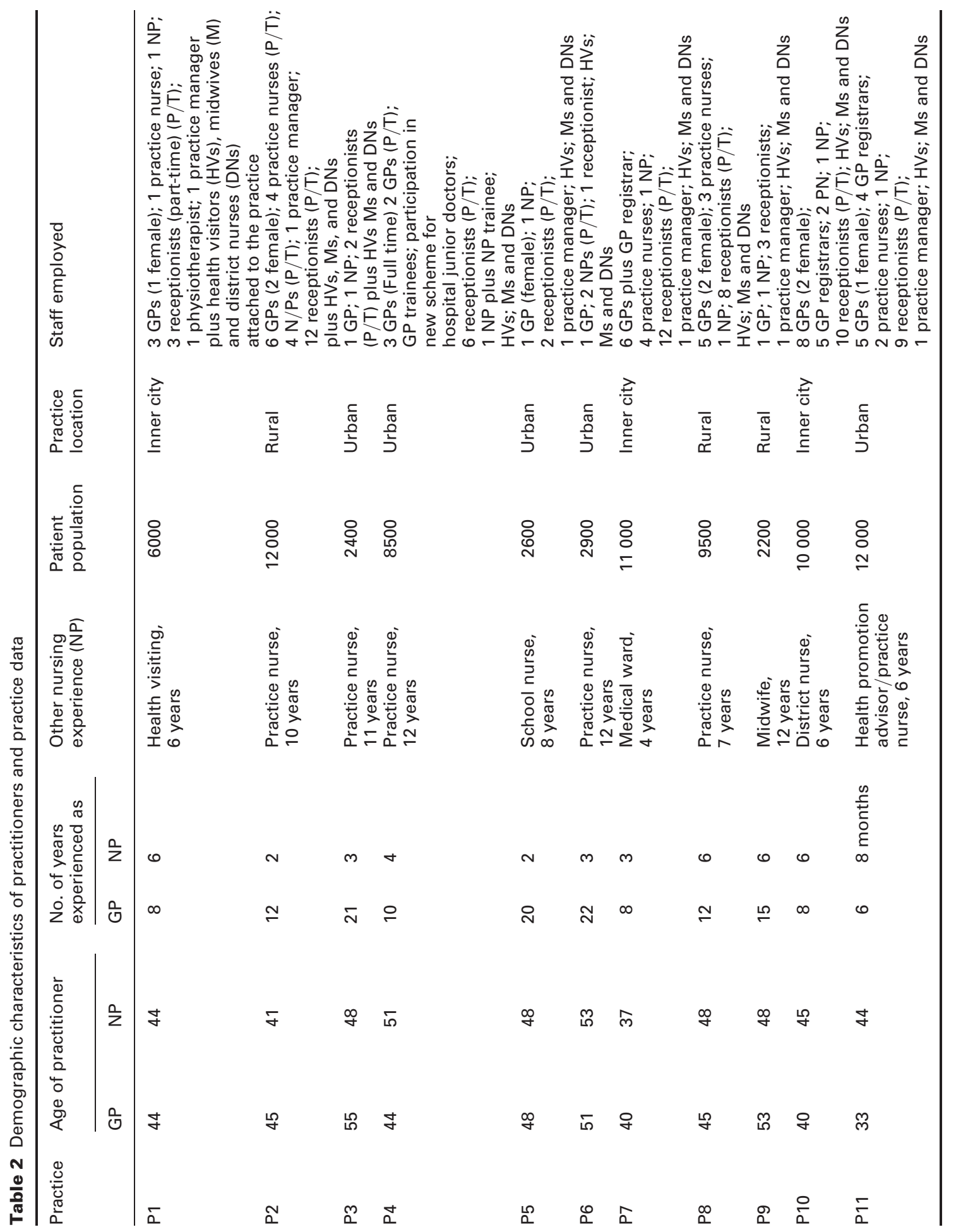


problem-solving moves in relation to their underpinning knowledge of the presenting problem.

Information Processing Theory (IPT) is influential in studies investigating nurses' decision-making, particularly since Elstein et al's (1978) seminal work on medical diagnostic decision-making. The theory has been used to explore and explain different aspects of nurse decision-making. A large number of the studies used simulated cases rather than real life clinical situations, although the use of the former have been criticized for their dissimilarity to the latter. Greenwood (2000) provides three reasons for the dissimilarity: the amount of information available to the participant is controlled, information is presented serially and the motivations and goals of the participants are presumed to be singular and simple. A counter argument to this view relates to whether or not, from both the patient's and an ethical perspective, it is advisable to permit 'think aloud' procedures during real clinical situations. Greenwood's (2000) views are rejected in this study for the following reasons: (i) some patients in the real clinical setting provide information serially as well as in a controlled manner, particularly if they are inarticulate. The skill of obtaining the relevant verbal information lies with the doctor or nurse, (ii) by complementing the 'think aloud' process with questioning at the end of the procedure, the motivations and goals of the participants become explicit. The use of the theory for this study is based on the proposition that:

The protocol is a record of the subject's ongoing behaviour, and an utterance at time $t$ is taken to indicate knowledge or operation at time $t$. Retrospective accounts leave much more opportunity for the subject to mix current knowledge with past knowledge, making reliable inferences from the protocol difficult ... All theorizing about the causes and consequences of the subject's knowledge state is carried out and validated by the experimenters, not by the subject.

Newell and Simon, 1972: 184

In the absence of an educational benchmark for NPs, the break down of these components in the decision-making process is useful for understand- ing the knowledge structures for the clinical problems they encounter. Understanding one's errors using this framework is a useful way to learn how to correct faulty reasoning. IPT allows the diagnostic process to be explicit, aiding a systematic way of teaching. A process that is understood can be taught. The use of the framework along with the demographic details of the practitioners (Table 2), revealed that diagnostic performance depended on clinical experience in a particular domain or area of specialization. This consists of several aspects: accumulated knowledge, skill in data collection, skill in performing physical examination and skill in diagnostic reasoning. The relative weights of each of these aspects in contributing to the overall diagnostic performance may differ according to the presenting problem and may differ with each practitioner. The framework was useful in highlighting the similarities in knowledge structures of the participants' responses, irrespective of whether they were right or wrong. However, not surprisingly, NPs' problem-solving skills tended to decrease in unfamiliar areas possibly due to a restriction of cases referred for consultation. The scenarios in this study were ones deemed suitable for consultation by either practitioner.

\section{Conclusions}

Expert knowledge in medicine, like nursing, is highly complex and many different schemata are used by experts to help organize and explain patient data. The production of an appropriate judgement and decision may come from a pattern matching process against schema templates. This process is a result of repeated experience with patients, as well as from formal educational input. Thus the solution of a patient problem is dependent upon how the problem presents itself, and the body of knowledge held by the practitioner.

Where commonalities exist between GPs and NPs, it is argued that both groups attended to the same salient points in the data. It seemed that both groups had learned how to classify problems with respect to the type of solution required: for example, pattern matching, algorithms and information processing methods. For some NPs, the problems in their judgement and decision-making seem to arise at the identification and elaboration 
knowledge stage of problem-solving because of a lack of experience of, and exposure to, the condition in question.

This study demonstrates that there is convergent validity between the NPs and GPs who gave correct responses to the scenarios. It concludes that where NPs are given the opportunity to undertake tasks that may be deemed suitable for consultation by either practitioner, they are as likely to achieve a correct diagnosis and offer similar treatment as their GP counterpart. However, which cases might be deemed suitable for NP consultation, currently remains the decision of the individual GP.

\section{References}

Baron, J. 1994: Thinking and deciding, 2nd edn. Cambridge: Cambridge University Press.

Elstein, A.S., Shulman, L.S. and Sprafka, S.A. 1978: Medical problem solving: an analysis of clinical reasoning. Cambridge, MA: Harvard University Press.

Feltovich, P.J. and Barrows, H.S. 1984: Issues of generality in medical problem solving. In Schmidt, H.G and De Volder, M.L., editors, Tutorials in problem-based learning: a new direction in teaching health professions. Assen, Netherlands: Van Gorcum, 128-42.

Gale, J. and Marsden, P. 1985: Diagnosis: process not product. In Sheldon, M., Brooke, J. and Rector, A., editors, Decisionmaking in general practice. London: Macmillan, 59-90.

Greenwood, J. 2000: Critical thinking and nursing scripts: the case for the development of both. Journal of Advanced Nursing 31, 428-36.

Johnson, P.E. 1983: What kind of an expert should a system be? Journal of Medicine and Philosophy 8, 77-97.

Joseph, G.M. and Patel, V. 1990: Domain knowledge and hypothesis generation in diagnostic reasoning. Medical Decision Making 10, 31-46.
Kahneman, D., Slovic, P. and Tversky, A. editors, 1982: Judgement under uncertainty: heuristics and biases. Cambridge: Cambridge University Press.

Marshall, S.P. 1995: Schemas in problem solving. Cambridge: Cambridge University Press.

McCormick, A., Fleming, D. and Charlton, J. 1995: Morbidity statistics from general practice: fourth national study 1991-1992. Series MB5 No.3. A study carried out by the Royal College of General Practitioners, the Office of Population Censuses and Surveys, and the Department of Health. London: Her Majesty's Stationery Office.

Minsky, M. 1975: A framework for representing knowledge. In Winston, P., editor, The psychology of computer vision. New York: McGraw-Hill, 211-18.

Newell, A. and Simon, HA. 1972: Human problem solving. Englewood Cliffs, NJ: Prentice Hall.

Norman, G.R. and Tugwell, P. 1982: A comparison of resident performance on real and simulated patients. Journal of Medical Education 19, 43-47.

Offredy, M. 2002: Decision making in primary care: outcomes from a study using patient scenarios. Journal of Advanced Nursing 40, 532-541.

Parrino, T.A. and Mitchell, R. 1989: Diagnosis as a skill: a clinical perspective. Perspectives in Biology and Medicine 33, 19-44.

Ramsay, L.E., Williams, B., Johnston, G.D., MacGregor, G.A., Poston, L., Potter, J.F., Poulter, N.R. and Russel, G. 1999: BHS guidelines: guidelines for management of hypertension: report of the third working party of the British Hypertension Society. Journal of Human Hypertension 13, 569-92.

Reason, J. 1990: Human error. Cambridge: Cambridge University Press.

Rumelhart, D. 1975: Notes on a schema for stories. In Bobrow, D. and Collins, A., editors, Representation and understanding. New York: Academic Press, 237-72.

Schank, R.C. 1975: The structure of episodes in memory. In Bobrow, D. and Collins, A., editors, Representation and understanding. New York: Academic Press, 211-36.

Schank, R.C. and Abelson, R.P. 1977: Scripts, plans, goals and understanding. Hillsdale, NJ: Lawrence Erlbaum.

Shanteau, J. 1992: How much information does an expert use? Is it relevant? Acta Psychology 81, 75-86. 